\title{
THE MASS PARAMETERS FOR THE AVERAGE MEAN-FIELD POTENTIAL ${ }^{\dagger}$
}

\author{
A. GÓŻDŻ*, K. POMORSKI*, M. BRACK and E. WERNER \\ Institute of Theoretical Physics, University of Regensburg, D-8400 Regensburg, West Germany \\ Received 10 December 1984 \\ (Revised 25 March 1985)
}

\begin{abstract}
Starting from a mean-field hamiltonian with pairing interaction, we use the generator coordinate method (GCM) and a generalized gaussian overlap approximation to derive a multidimensional collective hamiltonian for large-amplitude motion. Numerical calculations are performed for Nilsson and Woods-Saxon potentials with BCS pairing. The BCS wave function is taken as the generator function and the deformation parameters of the single-particle mean field are used as the generator coordinates. We find that the GCM mass parameters on the average are smaller than those of the cranking ( + BCS) model by a factor of $-\frac{2}{3}$. In the present approach, the zero-point energy correction to the collective potential is shown to vanish identically.
\end{abstract}

\section{Introduction}

The problem of the microscopic determination of collective mass parameters for a given mean-field potential has a rather long history. It is even difficult to quote all the papers devoted to this subject.

The main idea how to calculate the inertial parameters for nuclear rotation was proposed by Inglis and Belyaev in the so-called cranking model ${ }^{1}$ ). Soon it was successfully adapted and applied to nuclear vibration by Bès ${ }^{2}$ ). Such collective cranking masses were then used to calculate spontaneous fission lifetimes with Nilsson ${ }^{3}$ ) and Woods-Saxon potentials ${ }^{4}$ ) and to estimate nuclear quadrupole vibrations [e.g. ref. ${ }^{5}$ ) and references therein].

The cranking approach assumes that in the time-dependent Schrödinger equation the whole time dependence goes via the collective parameters. In the adiabatic approximation one obtains the expectation value of the energy as a sum of two terms corresponding to the kinetic and potential energies. The kinetic energy determines the collective mass tensor. A quantal collective hamiltonian is then obtained after a somewhat arbitrary quantization of the classical Hamilton equation ${ }^{6}$ ). This cranking procedure was often criticised because of this "second quantization", which is needed to derive a collective hamiltonian and the fact that it always starts from the

† Work supported in parts by GSI Darmstadt and Deutsche Forschungsgemeinschaft.

* On leave of absence from: Department of Theoretical Physics, University MCS, Lublin, Poland. 
time-dependent Schrödinger equation, while nuclear rotation and vibration are stationary processes.

The method proposed by Brink and Weiguny ${ }^{7}$ ) is free of these disadvantages. Using the generator coordinate method ${ }^{8,12}$ ) and the gaussian approximation for the overlap of generating functions, they derived a collective Schrödinger-type equation. This idea was used by several authors [see e.g. refs. $\left.{ }^{9-11}\right)$ ]. It is our aim to apply this concept to multidimensional shape vibrations and in particular to the fission mode. We derive the proper collective Schrödinger equation, starting from a mean-field potential depending on some collective parameters and including a residual pairing interaction. The main purpose of the present paper is to compare the standard cranking masses with those obtained by the generator coordinate method within the gaussian overlap approximation [see also refs. ${ }^{13,14}$ )].

The paper is organized as follows. In sect. 2 we repeat the main ideas of the generator coordinate method, the gaussian overlap approximation and the way to derive a collective hamiltonian. For simplicity, we restrict the presentation here to the case of one collective variable. In sect. 3 , the multidimensional case is treated using a covariant notation and including pairing interactions. Those readers who are not so much interested in formal details may jump sect. 3 and pass directly to sect. 4 where numerical results are discussed. Some technical details and more involved formulae are presented in the two appendices.

\section{Derivation of a collective hamiltonian (one-dimensional case)}

In the generator coordinate method (GCM) of Griffin, Hill and Wheeler ${ }^{8}$ ), one considers a family of many-particle wave functions $|a\rangle$ which depend parametrically on one or several collective variables $a_{i}(i=1,2, \ldots n)$. With the help of these so-called generating functions, an approximate wave function of the many-particle system is constructed by taking superpositions of the form

$$
|\Psi\rangle=\int \mathrm{d} a f(a)|a\rangle \text {. }
$$

(We treat here the case $n=1$; the multidimensional case is discussed in the next section.) The weight function $f(a)$ of the ansatz $(2.1)$ is determined from the variational principle for the expectation value of the total many-body hamiltonian $\hat{H}$,

$$
\delta \frac{\langle\Psi|\hat{H}| \Psi\rangle}{\langle\Psi \mid \Psi\rangle}=0,
$$

which leads to the Hill-Wheeler integral equation for $f(a)$ [refs. $\left.{ }^{8.15}\right)$ ]. Its solution is in general rather difficult. However, one can easily obtain a collective hamiltonian using the so-called gaussian overlap approximation (GOA) ${ }^{7}$ ), where one assumes the overlap integral $\left\langle a \mid a^{\prime}\right\rangle$ to be of gaussian form:

$$
\langle a \mid a\rangle \approx \exp \left\{-\frac{1}{2} \gamma(\xi)\left(a-a^{\prime}\right)^{2}\right\} \equiv \mathscr{N}\left(a, a^{\prime}\right),
$$


with $\xi \in\left(a, a^{\prime}\right)$. One then writes the expectation value of $\hat{H}$ as

$$
\langle\Psi|\hat{H}| \Psi\rangle=\int \mathrm{d} a \int \mathrm{d} a^{\prime} f^{*}(a) h\left(a, a^{\prime}\right)\left\langle a \mid a^{\prime}\right\rangle f\left(a^{\prime}\right),
$$

where

$$
h\left(a, a^{\prime}\right)=\frac{\langle a|\hat{H}| a\rangle}{\left\langle a \mid a^{\prime}\right\rangle}
$$

is a (reduced) energy overlap function. Next one defines a new metric by introducing the variable $\alpha$,

$$
\alpha=\int \sqrt{\frac{\gamma(q)}{\gamma_{0}}} \mathrm{~d} q \Rightarrow \mathrm{d} \alpha=\sqrt{\frac{\gamma(a)}{\gamma_{0}}} \mathrm{~d} a,
$$

so that the norm $\left\langle\alpha \mid \alpha^{\prime}\right\rangle$ becomes a gaussian with a constant width $\gamma_{0}$. Using

$$
\mathscr{N}\left(\alpha, \alpha^{\prime}\right)=\exp \left\{-\frac{1}{2} \gamma_{0}\left(\alpha-\alpha^{\prime}\right)^{2}\right\} \equiv \int_{-\infty}^{\infty} \mathrm{d} \xi \sqrt{\gamma_{0}} \mathscr{N}^{1 / 2}(\alpha, \xi) \mathscr{N}^{1 / 2}\left(\xi, \alpha^{\prime}\right)
$$

with

$$
\mathscr{N}^{1 / 2}(\alpha, \xi)=\left(\frac{2}{\pi}\right)^{1 / 4} \exp \left\{-\gamma_{0}(\alpha-\xi)^{2}\right\}
$$

one can now rewrite eq. (2.4) as

$$
\langle\Psi|\hat{H}| \Psi\rangle=\int \mathrm{d} \alpha \int \mathrm{d} \alpha^{\prime} \int \mathrm{d} \xi \sqrt{\gamma_{0}} \tilde{f}^{*}(\alpha) \mathscr{N}^{1 / 2}(\alpha, \xi) h\left(\alpha, \alpha^{\prime}\right) \mathscr{N}^{1 / 2}\left(\xi, \alpha^{\prime}\right) \tilde{f}\left(\alpha^{\prime}\right)
$$

The important step is now to Taylor-expand the function $h\left(\alpha, \alpha^{\prime}\right)$ around $\alpha=\alpha^{\prime}=\xi$ up to second order in $(\alpha-\xi)$ and $\left(\alpha^{\prime}-\xi\right)$. After some partial integrations the energy takes the form

$$
\langle\Psi|\hat{H}| \Psi\rangle=\int \mathrm{d} q \sqrt{\gamma(q)} \phi^{*}(q) \hat{\mathscr{H}}_{\text {coll }}\left(q, \frac{\partial}{\partial q}\right) \phi(q),
$$

where

$$
\phi(q)=\int \mathrm{d} a f(a) \mathscr{N}^{1 / 2}(a, q),
$$

and the collective hamiltonian $\hat{\mathscr{H}}_{\text {coll }}$ is defined by

$$
\hat{\mathscr{H}}_{\text {coll }}=-\frac{1}{\sqrt{\gamma(q)}} \frac{\partial}{\partial q} \sqrt{\gamma(q)} \frac{\hbar^{2}}{2 \mathscr{H}(q)} \frac{\partial}{\partial q}+V(q) .
$$

The inertial parameter $\mathscr{M}(q)$ is given by

$$
\mathscr{M}^{-1}(q)=\frac{1}{2(\gamma(q))^{2}}\left[\left(\frac{\partial^{2}}{\partial a \partial a^{\prime}}-\frac{\partial^{2}}{\partial a^{2}}+\frac{\partial \ln \gamma}{\partial q} \frac{\partial}{\partial a}\right) h\left(a, a^{\prime}\right)\right]_{a=a^{\prime}=q}
$$


The collective potential energy $V(q)$ is

$$
V(q)=\langle q|\hat{H}| q\rangle-\varepsilon_{0}(q)
$$

it contains besides the static expectation value of the hamiltonian $H$ also the zero-point energy correction $\varepsilon_{0}$ [refs. $\left.{ }^{9.11}\right)$ ] which has the form

$$
\varepsilon_{0}(q)=\frac{1}{2 \gamma(q)}\left[\left.\frac{\partial^{2} h\left(a, a^{\prime}\right)}{\partial a \partial a^{\prime}}\right|_{a=a^{\prime}=q}+\left\langle q\left|\frac{\partial \hat{H}}{\partial q} \frac{\partial}{\partial q}\right| q\right\rangle\right] .
$$

Note that the last terms in the square brackets of eqs. (2.13) and (2.15) are omitted in ref. ${ }^{15}$ ), although they only vanish in particular cases (see subsect. 3.3 below).

An extended discussion of the relation between the eigenvalues of $\hat{\mathscr{H}}_{\text {coll }}$ and those of the many-body hamiltonian $\hat{H}$ may be found in refs. ${ }^{12}$ ). It is important to remark also that the GOA method described above can be applied for any operator, not only for the hamiltonian, to obtain its representation in the collective space.

\section{Multidimensional case}

\subsection{THE OVERLAP FUNCTION}

We shall now discuss in detail the case of $n$ collective variables which we take to be the contravariant components of a $n$-dimensional vector in the collective space:

$$
a=\left(a^{1}, a^{2}, \ldots, a^{n}\right) \text {. }
$$

The corresponding many-particle wave function will again be denoted by $|a\rangle$.

The first step is to generalize the GOA. We take the overlap $\left\langle a \mid a^{\prime}\right\rangle$ to be of the following form ${ }^{16}$ ):

$$
\left\langle a \mid a^{\prime}\right\rangle \approx \exp \left\{-\frac{1}{2} \sum_{\kappa=1}^{n}\left[\Gamma^{\kappa}\left(a, a^{\prime}\right)\right]^{2}\right\} \equiv \mathscr{N}\left(a, a^{\prime}\right) .
$$

The following general conditions for the $\Gamma^{\kappa}$ should be satisfied for a fixed but arbitrary basic point $a_{0}$ and arbitrary points $a$ and $a^{\prime}$ :

(i) the jacobian $\frac{\partial\left(\alpha^{1}, \alpha^{2}, \ldots, \alpha^{n}\right)}{\partial\left(a^{1}, a^{2}, \ldots, a^{n}\right)} \neq 0$,

(ii) $\Gamma^{\kappa}\left(a, a_{0}\right)+\Gamma^{\kappa}\left(a_{0}, a^{\prime}\right)=\Gamma^{\kappa}\left(a, a^{\prime}\right)$,

(iii) $\Gamma^{\star}\left(a, a^{\prime}\right)=-\Gamma^{\star}\left(a^{\prime}, a\right)$.

Hereby we have defined the coordinate $\alpha^{\kappa}$ as

$$
\alpha^{\kappa}=\Gamma^{\kappa}\left(a, a_{0}\right), \quad \kappa=1,2, \ldots, n ;
$$

it defines a metric tensor which is a Kronecker $\delta$-function:

$$
\tilde{\gamma}_{\mu \nu}(\alpha)=\delta_{\mu \nu} .
$$

Thus, the exponent of the overlap function, eq. (3.2), is a scalar form in the collective 
space. Together with the conditions (3.5), the ansatz (3.2) seems to be flexible enough to represent a wide class of overlap functions.

For further convenience we define a scalar function $g(a)$ by the relation

$$
f(a)=\sqrt{\gamma(a)} g(a),
$$

where $\gamma(a)=\operatorname{det}\left(\gamma_{\mu \nu}(a)\right)$ and the matrix tensor $\gamma_{\mu \nu}(a)$ in the collective coordinates $\left\{a^{\kappa}\right\}$ is obtained from eq. (3.4) by the standard procedure

$$
\gamma_{\mu \nu}(a)=\sum_{\kappa=1}^{n} \frac{\partial \Gamma^{\kappa}\left(a, a_{0}\right)}{\partial a^{\mu}} \frac{\partial \Gamma^{\kappa}\left(a, a_{0}\right)}{\partial a^{\nu}}
$$

\subsection{THE COLLECTIVE HAMILTONIAN}

We now rewrite the energy overlap function (2.4) in the $\alpha$-coordinates:

$$
\langle\Psi|\hat{H}| \Psi\rangle=\iint \mathrm{d} \alpha \mathrm{d} \alpha^{\prime} g(\alpha)^{*} h\left(\alpha, \alpha^{\prime}\right) \mathcal{N}\left(\alpha, \alpha^{\prime}\right) g\left(\alpha^{\prime}\right),
$$

where the overlap function is of gaussian form

$$
\mathscr{N}\left(\alpha, \alpha^{\prime}\right)=\exp \left\{-\frac{1}{2} \sum_{\kappa=1}^{n}\left(\alpha^{\kappa}-\alpha^{\kappa \kappa}\right)^{2}\right\} .
$$

In the next step, in principle, we can replace $h\left(\alpha, \alpha^{\prime}\right)$ by an infinite series of differential operators which no longer depend on $\alpha$ and $\alpha^{\prime}$. However, for practical reasons we approximate $h\left(\alpha, \alpha^{\prime}\right)$ only by a second-order differential operator. To achieve this, we expand $h\left(\alpha, \alpha^{\prime}\right)$ around $\alpha=\alpha^{\prime}=\xi$ up to second order:

$$
\begin{aligned}
h\left(\alpha, \alpha^{\prime}\right)= & h(\xi, \xi)+\left(\frac{\partial h}{\partial \alpha^{\mu}}\right)_{\xi}\left(\alpha^{\mu}-\xi^{\mu}\right)+\left(\frac{\partial h}{\partial \alpha^{\prime \mu}}\right)_{\xi}\left(\alpha^{\mu}-\xi^{\mu}\right) \\
& +\frac{1}{2}\left\{\left(\frac{\partial^{2} h}{\partial \alpha^{\mu} \partial \alpha^{\nu}}\right)_{\xi}\left(\alpha^{\mu}-\xi^{\mu}\right)\left(\alpha^{\nu}-\xi^{\nu}\right)+2\left(\frac{\partial^{2} h}{\partial \alpha^{\mu} \partial \alpha^{\prime \nu}}\right)_{\xi}\left(\alpha^{\mu}-\xi^{\mu}\right)\left(\alpha^{\prime \nu}-\xi^{\nu}\right)\right. \\
& \left.+\left(\frac{\partial^{2} h}{\partial \alpha^{\prime \mu} \partial \alpha^{\prime \nu}}\right)_{\xi}\left(\alpha^{\prime \mu}-\xi^{\mu}\right)\left(\alpha^{\prime \nu}-\xi^{\nu}\right)\right\}
\end{aligned}
$$

We rewrite $\mathcal{N}\left(\alpha, \alpha^{\prime}\right)$ again as a product integral:

$$
\mathscr{N}\left(\alpha, \alpha^{\prime}\right)=\int_{-\infty}^{\infty} \mathrm{d} \xi \mathscr{N}^{1 / 2}(\alpha, \xi) \mathscr{N}^{1 / 2}\left(\xi, \alpha^{\prime}\right)
$$

where

$$
\mathscr{N}^{1 / 2}(\alpha, \xi)=\left(\frac{2}{\pi}\right)^{n / 4} \exp \left\{-\sum_{\kappa=1}^{n}\left(\alpha^{\kappa}-\xi^{\kappa}\right)^{2}\right\}
$$


Taking the first and the second derivative of $\mathscr{N}^{1 / 2}$ we get

$$
\begin{aligned}
\left(\alpha^{\mu}-\xi^{\mu}\right) \mathscr{N}^{1 / 2}(\alpha, \xi) & =\frac{1}{2} \tilde{\gamma}^{\mu \nu} \frac{\partial}{\partial \xi^{\nu}} \mathscr{N}^{1 / 2}(\alpha, \xi) \\
\left(\alpha^{\mu}-\xi^{\mu}\right)\left(\alpha^{\nu}-\xi^{\nu}\right) \mathscr{N}^{1 / 2}(\alpha, \xi) & =\left(\frac{1}{4} \tilde{\gamma}^{\mu \mu^{\prime}} \tilde{\gamma}^{\nu \nu^{\prime}} \frac{\partial^{2}}{\partial \xi^{\mu^{\prime}} \partial \xi^{\nu^{\prime}}}+\frac{1}{2} \tilde{\gamma}^{\mu \nu}\right) \mathscr{N}^{1 / 2}(\alpha, \xi) .
\end{aligned}
$$

The symbol of summation is omitted following Einstein's contraction rule. Substitution of eqs. (3.10), (3.11) and (3.13) into eq. (3.8) gives after partial integration

$$
\begin{aligned}
\langle\Psi|\hat{H}| \Psi\rangle=\int \mathrm{d} \xi \phi^{*}(\xi) & \left\{h(\xi, \xi)+\frac{\bar{\partial}}{\partial \xi^{\nu}} \frac{1}{2} \tilde{\gamma}^{\mu \nu}\left(\frac{\partial h}{\partial \alpha^{\mu}}\right)_{\xi}+\frac{1}{2} \tilde{\gamma}^{\mu \nu}\left(\frac{\partial h}{\partial \alpha^{\mu \mu}}\right)_{\xi} \frac{\vec{\partial}}{\partial \xi^{\nu}}\right. \\
+ & \frac{1}{2}\left[\left(\frac{\bar{\partial}^{2}}{\partial \xi^{\mu^{\prime}} \partial \xi^{\eta^{\prime}}} \frac{1}{4} \tilde{\gamma}^{\mu \mu^{\prime}} \tilde{\gamma}^{\nu \nu^{\prime}}+\frac{1}{2} \tilde{\gamma}^{\mu \nu}\right)\right. \\
& \times\left(\frac{\partial^{2} h}{\partial \alpha^{\mu} \partial \alpha^{\nu}}\right)_{\xi}+\frac{1}{2} \frac{\bar{\partial}}{\partial \xi^{\nu^{\prime}}} \tilde{\gamma}^{\nu \nu^{\prime}}\left(\frac{\partial^{2} h}{\partial \alpha^{\mu} \partial \alpha^{\nu \nu}}\right)_{\xi} \tilde{\gamma}^{\mu \mu^{\prime}} \frac{\vec{\partial}}{\partial \xi^{\mu^{\prime}}} \\
& \left.\left.+\left(\frac{\partial^{2} h}{\partial \alpha^{\prime \mu} \partial \alpha^{\prime \nu}}\right)_{\xi}\left(\frac{1}{4} \tilde{\gamma}^{\mu \mu^{\prime}} \tilde{\gamma}^{\nu \nu^{\prime}} \frac{\bar{\partial}^{2}}{\partial \xi^{\mu^{\prime}} \partial \xi^{\nu^{\prime}}}+\frac{1}{2} \tilde{\gamma}^{\mu \nu}\right)\right]\right\} \phi(\xi),
\end{aligned}
$$

where

$$
\phi(\xi)=\int \mathrm{d} \alpha g(\alpha) \mathscr{N}^{1 / 2}(\alpha, \xi)
$$

and $\vec{\partial} / \partial \xi(\bar{\partial} / \partial \xi)$ are operators acting on all functions being on the right (left) side. Assuming that the reduced energy overlap is a real function and neglecting its derivatives of higher order than two, we obtain after transformation to the collective variables $\left\{a^{\kappa}\right\}$ again the expression (2.10) for the energy. The collective hamiltonian $\mathscr{H}_{\text {coll }}(q, \partial / \partial q)$ now reads $(h \equiv 1)$

$$
\mathscr{H}_{\text {coll }}=-\frac{1}{2} \frac{1}{\sqrt{\gamma}} \frac{\partial}{\partial q^{\mu}} \sqrt{\gamma}\left(\mathscr{M}^{-1}\right)^{\mu \mu^{\prime}} \frac{\partial}{\partial q^{\mu^{\prime}}}+V(q)
$$

with the inverse mass tensor

$$
\left(\mathscr{M}^{-1}(q)\right)_{\mu \nu}=-\frac{1}{2}\left(\frac{\Delta}{\Delta a^{\mu}} \frac{\Delta h\left(a, a^{\prime}\right)}{\Delta a^{\nu}}-\frac{\Delta}{\Delta a^{\mu}} \frac{\Delta h\left(a, a^{\prime}\right)}{\Delta a^{\prime \nu}}\right)_{a=a^{\prime}=q} .
$$

The collective potential energy $V(q)$ is again given by eq. (2.14), where the zero-point energy $\varepsilon_{0}(q)$ is in covariant notation

$$
\varepsilon_{0}(q)=-\frac{1}{2} \gamma^{\mu \nu}\left\{\left(\frac{\Delta}{\Delta a^{\mu}} \frac{\Delta h}{\Delta a^{\nu}}\right)_{q}-\frac{\Delta}{\Delta q^{\mu}}\left[\left(\frac{\Delta h}{\Delta a^{\nu}}\right)_{q}\right]\right\}
$$


$\Delta / \Delta q^{\mu}$ are covariant derivatives and $\gamma^{\mu \nu}$ stand for the contravariant components of the metric tensor (3.7). It is useful to express the mass tensor and the zero-point energy in terms of matrix elements of the generator wave function.

These formulae are given here for the special case when all the first derivatives of $h$, i.e. $\left(\partial h / \partial a^{\mu}\right)_{q}$ and the matrix elements $\left\langle q\left|\partial / \partial q^{\mu}\right| q\right\rangle$, are equal to zero. In the general case they can be directly obtained from eqs. (3.17) and (3.18). After short calculations we obtain for the inverse mass tensor

$$
\left(\mathscr{M}^{-1}\right)_{\mu \nu}=\left\langle q\left|\frac{\bar{\partial}}{\partial q^{\mu}} \hat{H} \frac{\vec{\partial}}{\partial q^{\nu}}\right| q\right\rangle_{L}+\frac{1}{2}\left\langle q\left|\frac{\bar{\partial}}{\partial q^{\nu}} \frac{\partial \hat{H}}{\partial q^{\mu}}\right| q\right\rangle .
$$

The so-called linked matrix element (with index $\mathrm{L}$ ) is hereby defined as ${ }^{15}$ )

$$
\left\langle q\left|\frac{\bar{\partial}}{\partial q^{\mu}} \hat{H} \frac{\vec{\partial}}{\partial q^{\nu}}\right| q\right\rangle_{\mathrm{L}} \equiv\left\langle q\left|\frac{\bar{\partial}}{\partial q^{\mu}} \hat{H} \frac{\vec{\partial}}{\partial q^{\nu}}\right| q\right\rangle-\left\langle q\left|\frac{\bar{\partial}}{\partial q^{\mu}} \frac{\vec{\partial}}{\partial q^{\nu}}\right| q\right\rangle\langle q|\hat{H}| q\rangle .
$$

The expression for the zero-point energy (3.18) can be simplified to

$$
\varepsilon_{0}(q)=\frac{1}{2} \gamma^{\mu \nu}\left(\mathscr{M}^{-1}(q)\right)_{\mu \nu}+\frac{1}{4} \gamma^{\mu \nu}\left\langle q\left|\frac{\vec{\partial}}{\partial q^{\nu}} \frac{\partial \hat{H}}{\partial q^{\mu}}\right| q\right\rangle
$$

For completeness we write down the approximate formula for the metric tensor

$$
\gamma_{\mu \nu} \approx\left\langle q\left|\frac{\vec{\partial}}{\partial q^{\mu}} \frac{\bar{\partial}}{\partial q^{\nu}}\right| q\right\rangle,
$$

which can be easily derived ${ }^{16}$ ) from

$$
\begin{aligned}
\gamma_{\mu \nu} & =-\left(\frac{\partial}{\partial s^{\mu}} \frac{\partial}{\partial s^{\nu}} \mathcal{N}\left(q+\frac{1}{2} s, q-\frac{1}{2} s\right)\right)_{s=0} \\
& \approx-\left(\frac{\partial}{\partial s^{\mu}} \frac{\partial}{\partial s^{\nu}}\left\langle q+\frac{1}{2} s \mid q-\frac{1}{2} s\right\rangle\right)_{s=0} .
\end{aligned}
$$

In the above equations, $\hat{H}$ is in principle the true many-body hamiltonian which does not depend on the collective parameters $a^{\mu}$. Then the second terms on the r.h.s. in eq. (3.19) and (3.21) vanish trivially, leading to the formulae usually quoted in the literature [see e.g. ref. ${ }^{15}$ )]. However, in practice one often chooses $\hat{H}$ to contain a phenomenological, deformation-dependent mean field such as a Nilsson model or a Woods-Saxon potential. In this case the matrix elements containing $\partial \hat{H} / \partial q^{\mu}$ contribute to the inertia and to the zero-point energy. In fact, we will see in the next subsection that the two terms in eq. (3.21) for $\varepsilon_{0}(q)$ then cancel exactly for a phenomenological free quasiparticle hamiltonian. 
3.3. MICROSCOPIC CALCULATION OF THE OVERLAP INTEGRALS FOR A MEAN-FIELD HAMILTONIAN WITH BCS PAIRING

In the following we shall derive the explicit microscopic expressions for the overlap integrals for the case of a phenomenological mean-field hamiltonian with pairing interaction in the BCS approximation. The mean field is taken to depend explicitly on the deformation parameters $a^{i}(i=1,2, \ldots n)$. It may be thought to be obtained in a Hartree-Fock calculation from an effective nucleon-nucleon interaction by the mean of external constraints ${ }^{\dagger}$. In our numerical calculation presented in sect. 4 , we have used phenomenological Nilsson and Woods-Saxon potentials. In any case our assumption is that the matrix elements of such a mean-field (+ BCS) hamiltonian represent those of the true many-body hamiltonian.

To simplify the presentation, we derive here all the formulae for one kind of particle, i.e. neutrons or protons. How to combine them to get the collective hamiltonian for the whole nucleus will be discussed in the next paragraph.

The generator function $|a\rangle$ is taken to be a BCS wave function

$$
|a\rangle=\prod_{\nu>0}\left(u_{\nu}+v_{\nu} c_{\nu}^{+} c_{-\nu}^{+}\right)|0\rangle .
$$

The BCS function is generated ${ }^{\dagger \dagger}$ by the sum of a single-particle hamiltonian $\hat{H}_{0}$ and a pairing hamiltonian $\hat{H}_{\text {pair }}$ :

$$
\hat{H}_{\mathrm{G}}=\hat{H}_{0}\left(a^{1}, a^{2}, \ldots, a^{n}\right)+\hat{H}_{\mathrm{pair}} .
$$

$H_{0}$ depends on the set of collective parameters $\left\{a^{i}\right\}$. We use first-order perturbation theory for the hamiltonian

$$
\hat{H}_{\mathrm{G}}^{\prime}=\hat{H}_{\mathrm{G}}+\frac{\partial \hat{H}_{\mathrm{G}}}{\partial a^{i}} \mathrm{~d} a^{i}
$$

in order to obtain the derivatives of the generator functions. In addition we assume that the BCS equations are fulfilled at each point of the collective space.

After some standard calculations one gets the following expression for the derivatives of $|a\rangle$ [refs. $\left.{ }^{2,4,17}\right)$ ]:

$$
\frac{\partial}{\partial a^{i}}|a\rangle \equiv \hat{P}_{i}|a\rangle=\sum_{\mu \nu}\left(\hat{P}_{i}\right)_{\mu \nu} \alpha_{\mu}^{+} \alpha_{-\nu}^{+}|a\rangle,
$$

where

$$
\begin{aligned}
\left(\hat{P}_{i}\right)_{\mu \nu} & =-\frac{\left\langle\mu\left|\partial \hat{H}_{0} / \partial a^{i}\right| \nu\right\rangle}{E_{\mu}+E_{\nu}}\left(u_{\mu} v_{\nu}+u_{\nu} v_{\mu}\right)-\delta_{\mu \nu}\left(G_{i}\right)_{\nu}, \\
\left(G_{i}\right)_{\nu} & =\frac{1}{2}\left(\frac{\Delta}{E_{\mu}^{2}} \frac{\partial \lambda}{\partial a^{i}}+\frac{e_{\mu}-\lambda}{E_{\mu}^{2}} \frac{\partial \Delta}{\partial a^{i}}\right)
\end{aligned}
$$

+ See also the discussion in appendix $B$.

${ }^{++}$To avoid confusion, we stress that $\hat{H}_{\mathrm{G}}$ is used only for constructing the generator functions and has in principle nothing to do with the true many-body hamiltonian $\hat{H}$ appearing in subsect. 3.2 . 
the derivatives of $\lambda$ and $\Delta$ with respect to the deformation parameters $a^{i}$ are given explicitly in appendix A. Having the expression for the infinitesimal translation operator $\hat{P}_{i}$, it is easy to calculate the width $\gamma_{i j}$ of the overlap $\left\langle a \mid a^{\prime}\right\rangle$ of the generator functions:

$$
\begin{aligned}
\gamma_{i j}(a) & =\left\langle a\left|\frac{\grave{\partial}}{\partial a^{i}} \frac{\vec{\partial}}{\partial a^{j}}\right| a\right\rangle=\left\langle a\left|\hat{P}_{i} \hat{P}_{j}\right| a\right\rangle \\
& =\sum_{\nu \mu}\left(\hat{P}_{i}\right)_{\nu \mu}^{*}\left(\hat{P}_{j}\right)_{\mu \nu} .
\end{aligned}
$$

The next step is to find the expansion coefficients of the reduced energy overlap $h\left(a, a^{\prime}\right)$. In order to do so, we assume that the noncollective excitation of the true many-body hamiltonian are approximated by a free quasiparticle $\mathrm{BCS}$ hamiltonian:

$$
\hat{H}=\hat{H}_{\mathrm{q} . \mathrm{p} .}=E_{\mathrm{BCS}}+\sum_{\nu>0} E_{\nu}\left(\alpha_{\nu}^{+} \alpha_{\nu}+\alpha_{-\nu}^{+} \alpha_{-\nu}^{+}\right)
$$

where

$$
E_{\mathrm{BCS}}=\sum_{\nu} 2 e_{\nu} v_{\nu}^{2}-\Delta^{2} / G-G \sum_{\nu} v_{\nu}^{4}
$$

The usual procedure ${ }^{2}$ ) is to use a subtracted effective hamiltonian:

$$
\hat{H}_{\mathrm{eff}}=\hat{H}_{\mathrm{G}}-\lambda(\hat{N}-N)=\hat{H}_{0}\left(a^{i}\right)-\lambda(\hat{N}-N)+\hat{H}_{\mathrm{pair}}
$$

which corresponds to $\hat{H}_{\text {q.p. }}$ after neglecting the four quasiparticle terms as frequently done in BCS calculations. The substraction of the particle-number operator from the single-particle hamiltonian is a very important procedure: it has to be applied when one works with BCS functions which conserve the particle number only on the average and guarantees that the mass parameters are independent of the zero of the single particle energy scale.

The zero-order expansion coefficient in (3.10) is simply equal to the BCS groundstate energy $E_{\mathrm{BCS}},(3.32)$. It has to be corrected later on by the Strutinsky procedure in order to give a good approximation to the true ground-state energy ${ }^{4}$ ). The first-order expansion coefficients of $h\left(a, a^{\prime}\right)$ vanish,

$$
\left\langle a\left|\frac{\overleftarrow{\partial}}{\partial a^{i}} \hat{H}\right| a\right\rangle=\left\langle a\left|\hat{H} \frac{\vec{\partial}}{\partial a^{i}}\right| a\right\rangle=\left\langle a\left|\hat{H}_{\mathrm{eff}} \hat{P}_{i}\right| a\right\rangle=0,
$$

because no two-quasiparticle term exists in the hamiltonian $\hat{H}_{\text {q.p: }}$ (Such a term vanishes after the Bogolubov-Valatin transformation of the hamiltonian $\hat{H}_{\text {eff }}$.) The first non-trivial terms are the second-order coefficients in the expansion (3.10):

$$
\begin{aligned}
h_{i j} & =\left\langle a\left|\frac{\bar{\partial}}{\partial a^{i}} \hat{H}_{\mathrm{eff}} \frac{\vec{\partial}}{\partial a^{j}}\right| a\right\rangle_{\mathrm{L}}=\sum_{\nu \mu}\left(P_{i}\right)_{\nu \mu}^{*}\left(E_{\nu}+E_{\mu}\right)\left(P_{j}\right)_{\mu \nu} \\
& =\sum_{\nu \mu}\left(\hat{H}_{0, i}\right)_{\nu \mu}\left(\hat{H}_{0, j}\right)_{\mu \nu}\left(E_{\nu}+E_{\mu}\right)^{-1}\left(u_{\nu} v_{\mu}+u_{\mu} v_{\nu}\right)^{2}+F_{i j}^{(1)},
\end{aligned}
$$


where

$$
\left(\hat{H}_{0, i}\right)_{\nu \mu} \equiv\left\langle\nu\left|\frac{\partial \hat{H}_{0}}{\partial a^{i}}\right| \mu\right\rangle .
$$

The first term on the r.h.s. of eq. (3.35) gives the leading contribution. The remaining terms, contained in $F_{i j}^{(1)}$ and shown explicitly in appendix A, give generally rather small contributions; they are due to the fact that the pairing parameters $\Delta$ and $\lambda$ depend on the collective parameters, as seen from eq. (3.29). Note that the denominator of the leading term contains the first power of the excitation energy $\Delta E=E_{\mu}+E_{v}$. Therefore this expression converges only slowly upon summing higher and higher two-quasiparticle excitations. In fact, convergence is only reached due to the decreasing absolute values of the matrix elements in the numerator.

It is important to note that when using the effective hamiltonian $\hat{H}_{\text {eff }}$, eq. (3.33), instead of the true many-body hamiltonian, we obtain the following relation,

$$
\left\langle a\left|\left(\frac{\partial \hat{H}_{\mathrm{eff}}}{\partial a^{i}}\right) \frac{\vec{\partial}}{\partial a^{j}}\right| a\right\rangle=-\left\langle a\left|\frac{\bar{\partial}}{\partial a^{i}} \hat{H}_{\mathrm{eff}} \frac{\vec{\partial}}{\partial a^{j}}\right| a\right\rangle_{\mathrm{L}}=-h_{i j},
$$

which together with eqs. (3.19) and (3.21) gives

$$
\begin{aligned}
\left(\mathscr{M}^{-1}\right)^{i l} & =\frac{1}{2} \gamma^{i j} h_{j k} \gamma^{k l}, \\
\varepsilon_{0}(q) & =0 .
\end{aligned}
$$

The two terms in the zero-point energy $\varepsilon_{0}(q)$, eq. (3.12), thus cancel identically, and the collective potential energy is simply equal to the (deformation-dependent) BCS ground-state energy ${ }^{\dagger}$. Eq. (3.37) for the mass parameter is essentially equivalent to that of refs. ${ }^{13,14}$ ) for the one-dimensional case.

In order to complete the microscopic formulae used in this paper, we give the expression for the cranking-model mass parameters ${ }^{4,17}$ ):

$$
B_{i j}=2 \sum_{\nu \mu}\left(\hat{P}_{i}\right)_{\nu \mu}^{*}\left(E_{\nu}+E_{\mu}\right)^{-1}\left(\hat{P}_{j}\right)_{\mu \nu}
$$

It leads to an expression similar to eq. (3.35) with, however, two more powers of the energies in the denominators (see appendix A). Therefore the cranking masses $B_{i j}$ converge much more rapidly than the GCM masses.

It is often useful to have simple estimates for the above microscopic quantities which can only be calculated numerically. For that purpose, we assume only diagonal matrix elements $\left(\hat{P}_{i}\right)_{\mu \mu}$ to contribute and neglect all terms containing $\partial \Delta / \partial a^{i}$ and $\partial \lambda / \partial a^{i}$. We then get

$$
\left\langle a\left|\frac{\bar{\partial}}{\partial a^{i}} \hat{H} \frac{\vec{\partial}}{\partial a^{j}}\right| a\right\rangle_{L} \approx \sum_{i j}^{(1)}, \quad \gamma_{i j} \approx \sum_{i j}^{(2)}, \quad B_{i j} \approx 2 \sum_{i j}^{(3)},
$$

\footnotetext{
+ The same result is, of course, also obtained in the case $\Delta \rightarrow 0$, i.e. using a simple phenomenological, deformed shell-model hamiltonian. (See appendix B for a more detailed discussion.)
} 
where for $K=1,2,3$

$$
\sum_{i j}^{(K)}=\sum_{\nu}\left(\hat{H}_{0, i}\right)_{\nu \nu}\left(\hat{H}_{0, j}\right)_{\nu \nu}\left(2 E_{\nu}\right)^{-K-2} \Delta^{2} .
$$

The sums $\Sigma_{i j}^{(K)}$ can be approximated on the average by the integrals ${ }^{4}$ )

$$
\sum_{i j}^{(K)} \approx \frac{\bar{Q}_{i j} \Delta^{2} \bar{g}}{2^{K}} \int_{\lambda-\Omega}^{\lambda+\Omega} \frac{\mathrm{d} e}{\left[(e-\lambda)^{2}+\Delta^{2}\right]^{(K+2) / 2}},
$$

with

$$
Q_{i j}=\left(\hat{H}_{0, i}\right)_{\nu \nu}\left(\hat{H}_{0, j}\right)_{\nu \nu} .
$$

Here $2 \Omega$ is the width of the pairing window and $\bar{g}=\bar{g}(\lambda)$ is the average s.p. level density at the Fermi energy $\lambda$. The integrals (3.42) can be easily evaluated and give the following estimates:

$$
h_{i j} \approx \bar{Q}_{i j} \bar{g}, \quad \gamma_{i j} \approx \frac{1}{8} \pi \bar{Q}_{i j} \bar{g} / \Delta, \quad B_{i j} \approx \frac{1}{3} \bar{Q}_{i j} \bar{g} / \Delta^{2} .
$$

In the one-dimensional case, or when the nondiagonal elements of the tensor $\gamma_{i j}$ vanish, the GCM mass parameter, eq. (3.37), is

$$
\mathscr{H}_{i i}=2\left(\gamma_{i i}\right)^{2} / h_{i i}
$$

and can be estimated as

$$
\mathscr{M}_{i i} \approx \frac{1}{32} \pi^{2} \bar{Q}_{i i} \bar{g} / \Delta^{2} \text {. }
$$

We see that the GCM mass parameter $\mathscr{M}_{i i}$ as well as the cranking mass $B_{i i}$ depend strongly on the pairing energy gap $\Delta$. It is interesting also that within the approximations (3.41) and (3.42), the ratio of the two mass parameters is constant

$$
\mathscr{M}_{i i} / B_{i i}=\frac{3}{32} \pi^{2} \approx 0.925 \text {. }
$$

We would like to stress here that the "diagonal matrix element approximation" works especially well for $B_{i i}$ because of the power 3 in the denominator (see eqs (3.39)-(3.41)). The effect of the nondiagonal matrix elements is rather nonnegligible for $\left\langle a\left|\hat{P}_{i} \hat{H} \hat{P}_{j}\right| a\right\rangle$, eq. (3.25), and because of the relation (3.44) we can expect that the mass $\mathscr{M}_{i i}$ will be even smaller than the estimate (3.45).

On the other hand, when the most important two-quasiparticle excitations have approximately the same energy $2 E$, another approximation can be made,

$$
\begin{aligned}
& h_{i j} \approx 2 E \sum_{\nu \mu}\left(\hat{P}_{i}\right)_{\nu \mu}^{*}\left(\hat{P}_{j}\right)_{\mu \nu}, \\
& \gamma_{i j} \approx \sum_{\nu \mu}\left(\hat{P}_{i}\right)_{\nu \mu}^{*}\left(\hat{P}_{j}\right)_{\mu \nu}, \\
& B_{i j} \approx \frac{1}{E} \sum_{\nu \mu}\left(\hat{P}_{i}\right)_{\nu \mu}^{*}\left(\hat{P}_{j}\right)_{\mu \nu},
\end{aligned}
$$


and one obtains in the case $\gamma_{i j}=\gamma_{i \kappa} \delta_{j}^{\kappa}$

$$
\mathscr{M}_{i i}=2\left(\gamma_{i i}\right)^{2} / h_{i i}=B_{i i},
$$

i.e. both masses become equal. Such a situation corresponds approximately to the calculation recently done for doubly-magic nuclei ${ }^{14}$ ) or to cases with an extremely large pairing gap parameter $\Delta$ (as discussed in sect. 4).

\section{Results and discussion}

Our numerical calculations of GCM and cranking parameters were performed for a Nilsson as well as a Woods-Saxon potential. The standard parameter set for these potentials was used ${ }^{18.4}$ ).

Calculations with the Nilsson potential were performed for the whole actinide region, but we present only some illustrative examples. We have chosen two collective degrees of freedom: $\varepsilon_{24}=\left(\varepsilon, \varepsilon_{4}(\varepsilon)\right)$ corresponding to the path of minimal liquid drop energy when the nucleus elongates, and $\varepsilon_{35}=\left(\varepsilon_{3}, \varepsilon_{5}\left(\varepsilon, \varepsilon_{3}\right)\right)$ as the most preferable mixture of $\lambda=3$ and $\lambda=5$ multipolarities when the reflection asymmetry is included. For the pairing strengths we have used ${ }^{19}$ )

$$
G_{\mathrm{p}}=g_{\mathrm{p}} / Z^{2 / 3}, \quad G_{\mathrm{n}}=g_{\mathrm{n}} / N^{2 / 3}
$$

with

$$
g_{\mathrm{p}}=g_{\mathrm{n}}=0.29 \hbar \stackrel{\circ}{0}_{0}
$$

which correspond with a very good accuracy to the standard values of ref. ${ }^{18}$ ). The pairing window $2 \Omega$ includes $\sqrt{15 Z(N)}$ levels above and below the Fermi surface.

The width $\gamma_{i j}$ of the BCS function overlap were calculated according to eq. (3.30), the GCM mass parameters using eqs. (3.37) and (C.4), and the cranking inertias by the formula (3.39). Both microscopic masses for ${ }^{154} \mathrm{Sm}$ are plotted in fig. 1 as functions of the quadrupole deformation $\varepsilon$. The thick solid and dashed lines represent the full cranking inertia $B_{\varepsilon \varepsilon}$ and GCM mass parameter $M_{\varepsilon \varepsilon}$, respectively. The thin lines were obtained neglecting all terms containing the derivatives $\partial \lambda / \partial \varepsilon$ and $\partial \Delta / \partial \varepsilon$ in eq. (3.6), which are responsible for the coupling to the pairing vibration modes. We see that the GCM mass is about $\frac{2}{3}$ times smaller than the cranking mass. Both quantities exhibit a similar shell structure. The shell effect is especially pronounced near the minima $(\varepsilon \sim \pm 0.25)$ in the potential energy surface. Also, the effect of coupling with the pairing vibration terms, which is usually smaller than $5 \%$ of the collective mass, becomes larger (up to 30\%) when the shell effect is large.

Similar results are presented for ${ }^{240} \mathrm{Pu}$ in fig. 2 . In addition we plot here the parameter $\gamma_{\varepsilon_{24} \varepsilon_{24}}$ which enters the collective hamiltonian (3.16). These results are obtained along the symmetric $\left(\varepsilon_{35}=0\right)$ path to fission $\left(\varepsilon_{24}\right)$. 


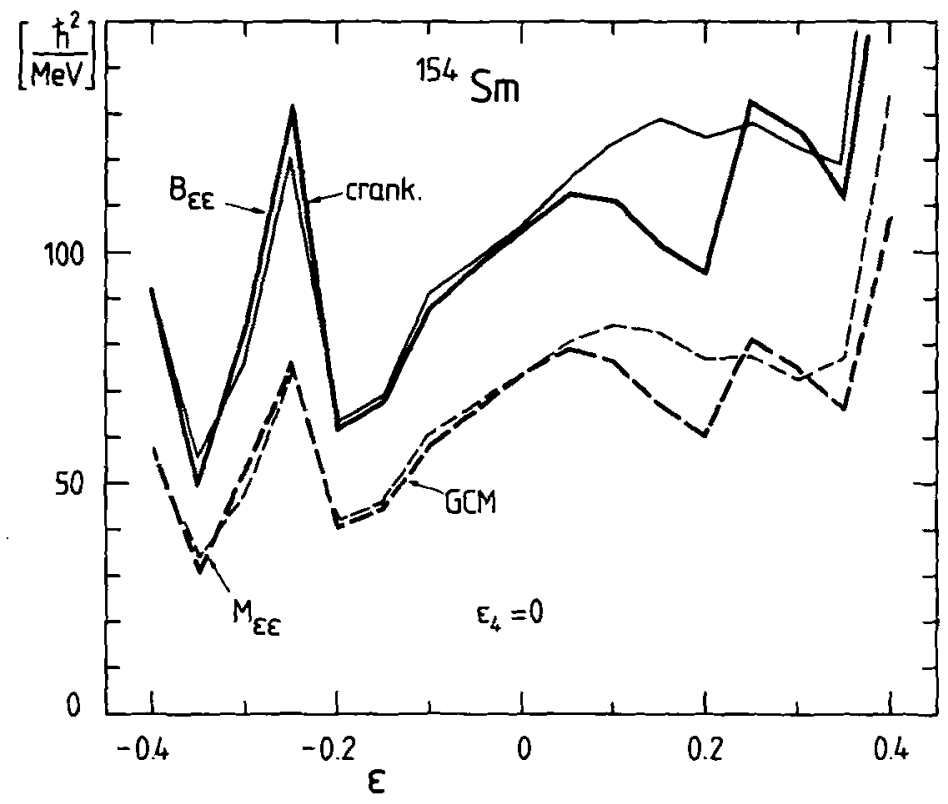

${ }^{154} \mathrm{Sm}$

Fig. 1. Cranking (solid lines) and GCM (dashed lines) masses for the quadrupole model ( $\varepsilon$ ) for ${ }^{154} \mathrm{Sm}$. The thin lines correspond to the case when the coupling to pairing vibrations is not taken into account.

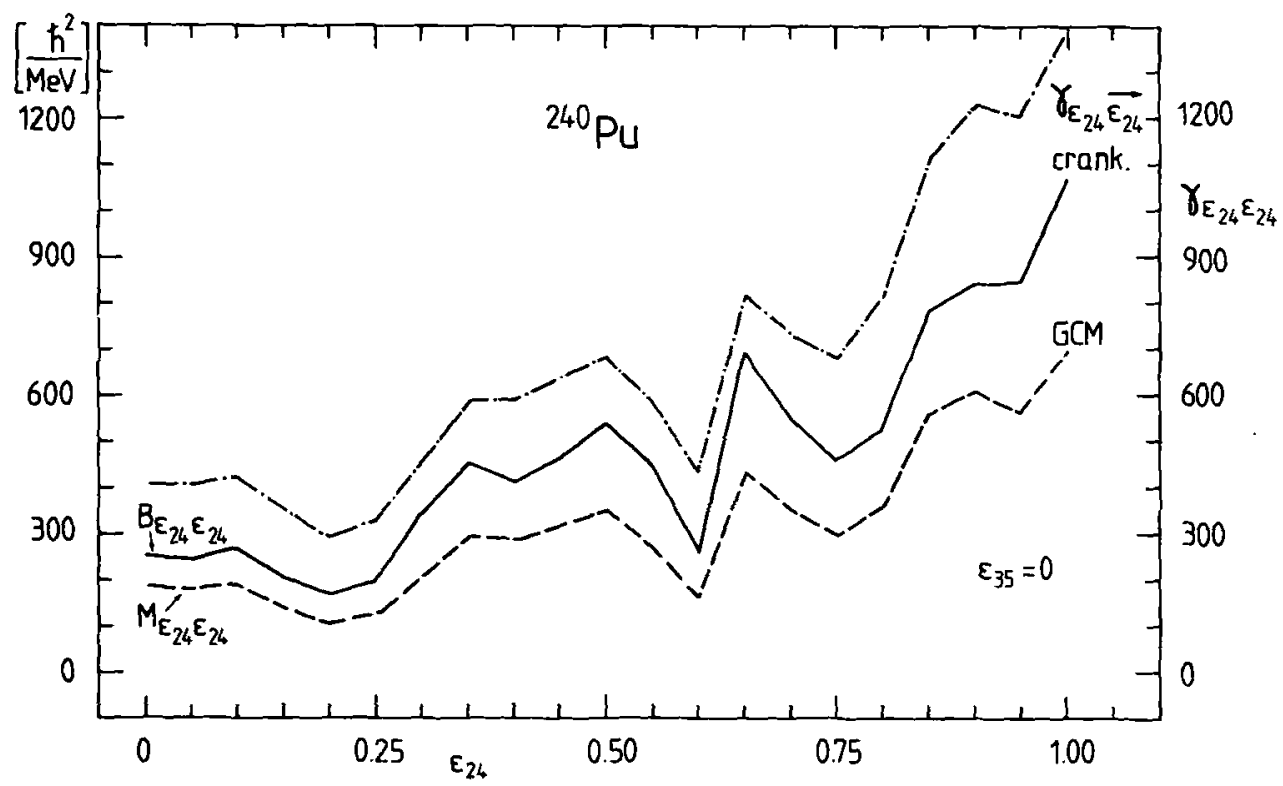

Fig. 2. Dependence of cranking (solid line) and GCM mass (dashed line) for elongation ( $\varepsilon_{24}$ ) and the width of the BCS function overlap $\gamma_{\varepsilon_{24} \varepsilon_{24}}$ (dashed-dotted line) for ${ }^{240} \mathrm{Pu}$. 


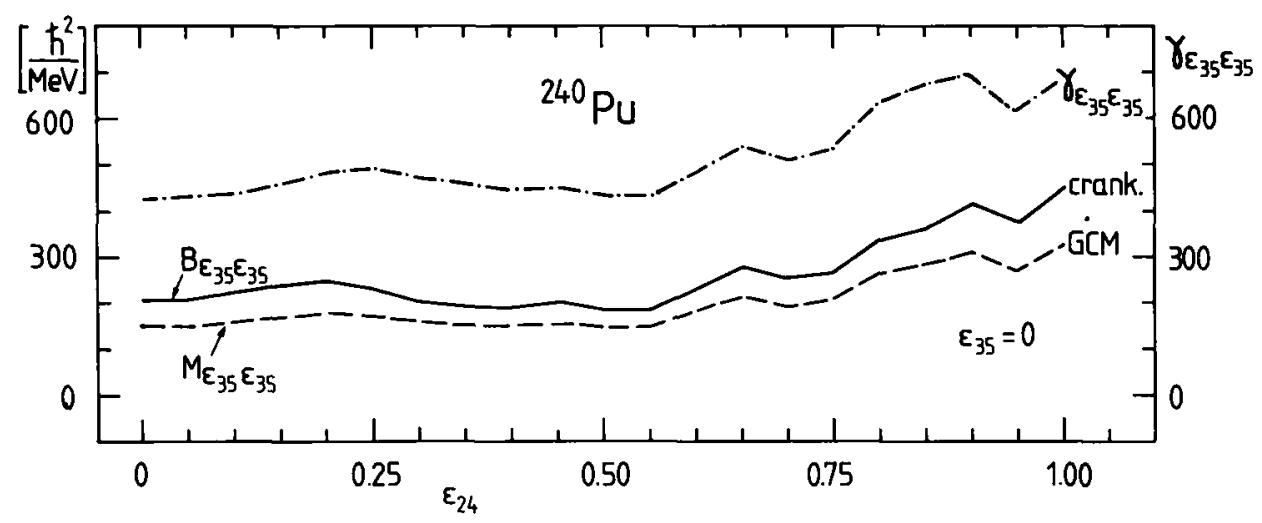

Fig. 3. The same as in fig. 2 , but for the left-right asymmetry mode $\left(\varepsilon_{35}\right)$.

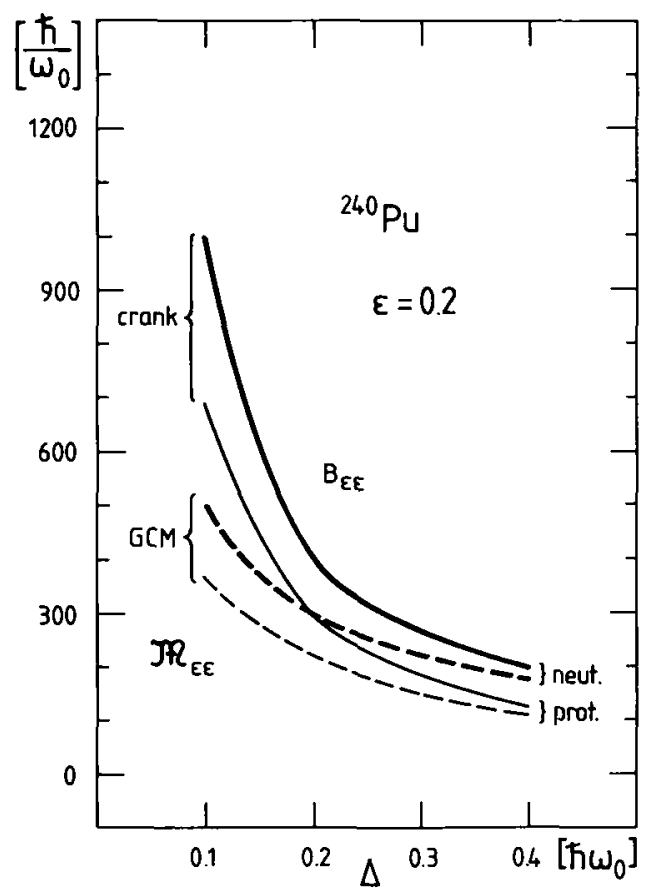

Fig. 4. Dependence of GCM and cranking masses for protons and neutrons in ${ }^{240} \mathrm{Pu}$ on the pairing gap $\Delta$. 
The results presented in figs. 1 and 2 are in line with the mass estimates done in ref. ${ }^{13}$ ) for the two-center model but contradict the conclusion of ref. ${ }^{14}$ ) that for nearly spherical configurations both cranking and GCM masses are the same. The conclusion of ref. ${ }^{14}$ ) is true only for the special case of the doubly-magic nucleus discussed there and when, furthermore, the diagonal matrix elements of $\hat{P}_{i}$, eq. (3.28), responsible for the coupling to the pairing vibration modes are neglected.

In fig. 3 the components of the $\gamma_{\varepsilon_{35} \varepsilon_{33}}, B_{\varepsilon_{33} \varepsilon_{35}}$ and $M_{\varepsilon_{33} \varepsilon_{35}}$ tensors corresponding to the left-right asymmetry mode are plotted along the same path to fission as in fig. 2. We see that in this case the shell structure is not so pronounced as in fig. 2 . This is mainly connected with the fact that for $\varepsilon_{35}=0$ the diagonal part of the operator $\hat{P}_{F_{13}}$ vanishes: $\left(\hat{P}_{e_{3 s}}\right)_{\nu \nu}=0$. Note that for the left-right asymmetry mode the GCM mass parameter is also smaller than the cranking inertia.

In sect. 3 we have made the general statement that the GCM and cranking inertias have to approach each other when the gap parameter $\Delta$ is increased. Fig. 4 illustrates this effect for the quadrupole components of the mass tensor.

The values of $h_{\varepsilon_{24} \varepsilon_{24}}, \gamma_{\varepsilon_{24} \varepsilon_{24}}$ and the cranking mass $B_{\varepsilon_{24} \varepsilon_{24}}$ are plotted in fig. 5 for a system with $Z=94$ protons. The solid lines represent the results of the exact calculation, while the dashed line corresponds to the values obtained using only the diagonal matrix elements in eq. (3.28). In the case of parameter $h_{\varepsilon_{24} \varepsilon_{24}}$, the sum of

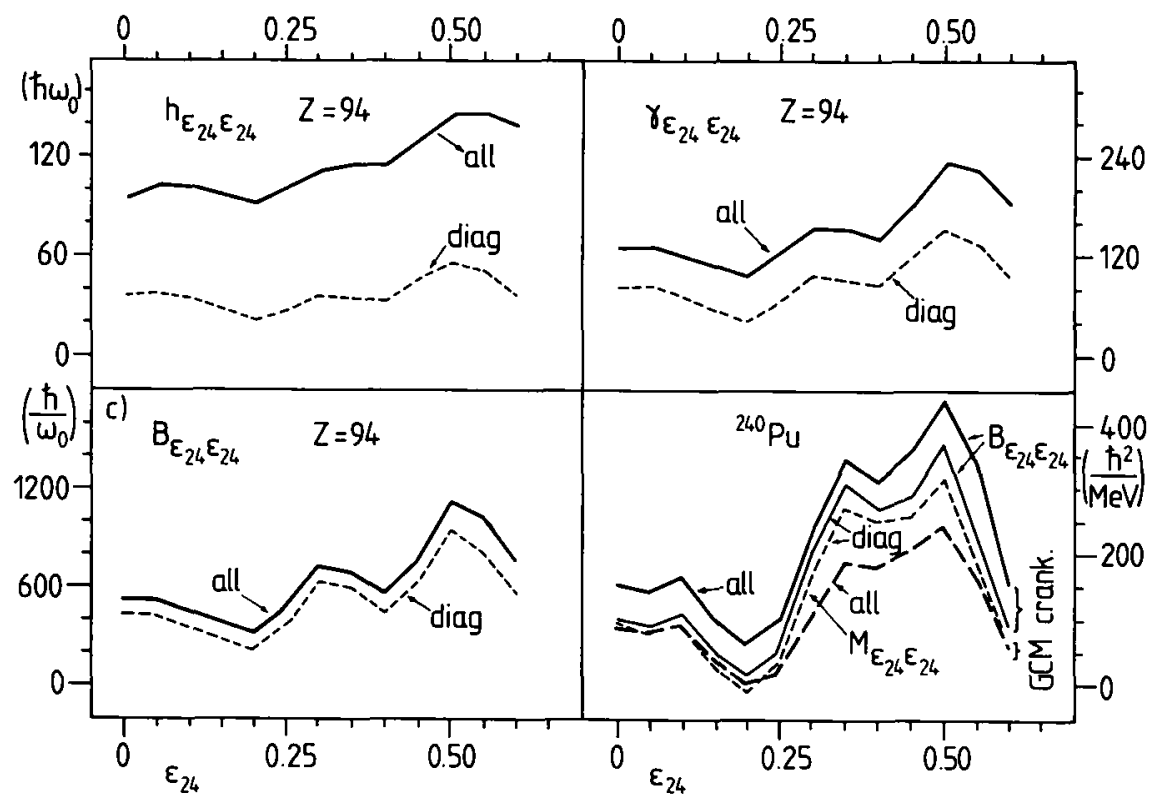

Fig. 5. Dependence of $h_{E_{24} \varepsilon_{24}}, \gamma_{e_{24} \varepsilon_{34}}$ and $B_{\varepsilon_{24} \varepsilon_{24}}$ for $Z=94$ protons and $\operatorname{GCM}\left(M_{\varepsilon_{24} \varepsilon_{24}}\right)$ and cranking $\left(B_{\varepsilon_{24} e_{24}}\right.$ ) masses for ${ }^{240} \mathrm{Pu}$ on the elongation $\varepsilon_{24}$. In the first three figures, the solid lines represent their complete values while the dashed lines correspond to the diagonal matrix elements approximation. 
the diagonal matrix elements contains on the average only around $\frac{1}{3}$ of the total sum (3.35), while for $\gamma_{\varepsilon_{24} \varepsilon_{24}}$ it gives already $\frac{2}{3}$ and for $B_{\varepsilon_{23} \varepsilon_{24}}$ even $\frac{5}{6}$ of the values. In the lower right of fig. 5 we plot for ${ }^{240} \mathrm{Pu}$ the full cranking mass (solid thick line) and that part which comes from the diagonal matrix elements only (thin solid line); the thick dashed line represents the whole GCM inertia, while the thin dashed line corresponds to the diagonal matrix element approximation. It is exciting that these approximate masses agree rather well with the crude estimate (3.46).

Looking at fig. 5 one also reaches the conclusion that both masses have approached each other when the size of the pairing window is decreased. In order to prove that this conclusion is generally true and is not only a special feature of the Nilsson potential, we performed a calculation with the Woods-Saxon potential for the same nucleus ${ }^{240} \mathrm{Pu}$ in the configuration corresponding to the second saddle. Two degrees of freedom, elongation (c) and left-right asymmetry $(\alpha)$, are taken into account ${ }^{4}$ ). We assumed the same gaps for protons and neutrons, $\Delta_{p}=\Delta_{n}=1 \mathrm{MeV}$, and varied the pairing window width $2 \Omega$ only. The results for three components of

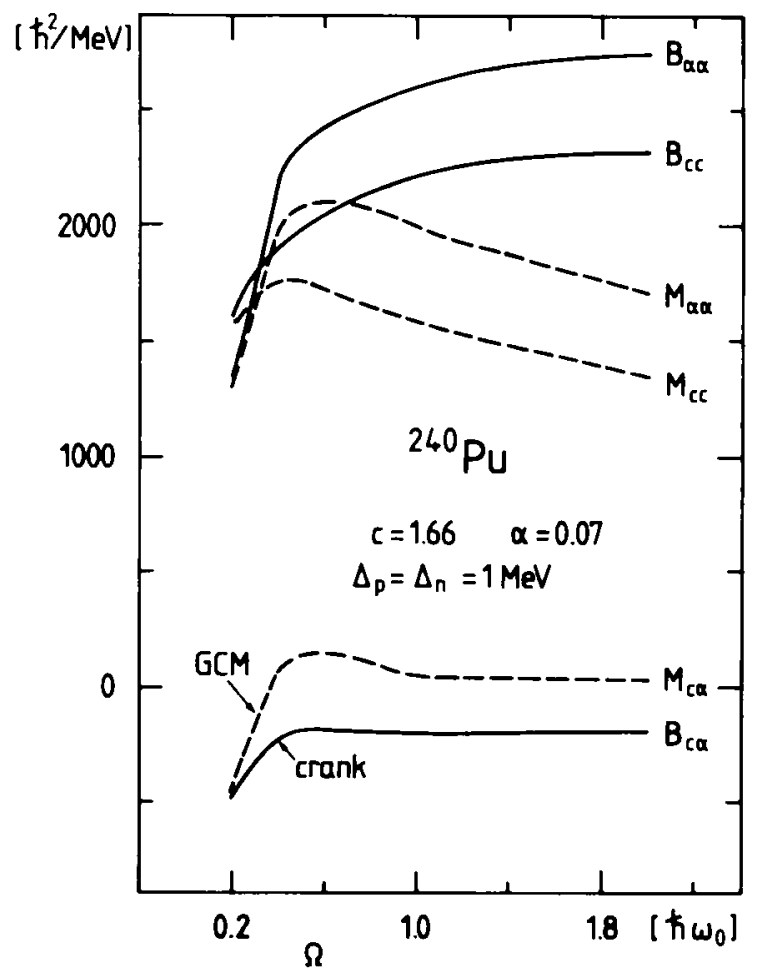

Fig. 6. Dependence of the elongation (c) and left-right asymmetry $(\alpha)$ components of the GCM mass tensor on the width $(2 \Omega)$ of the pairing window for $\Delta_{P}=\Delta_{n}=1 \mathrm{MeV}$. This diagram is obtained with a Woods-Saxon potential. 


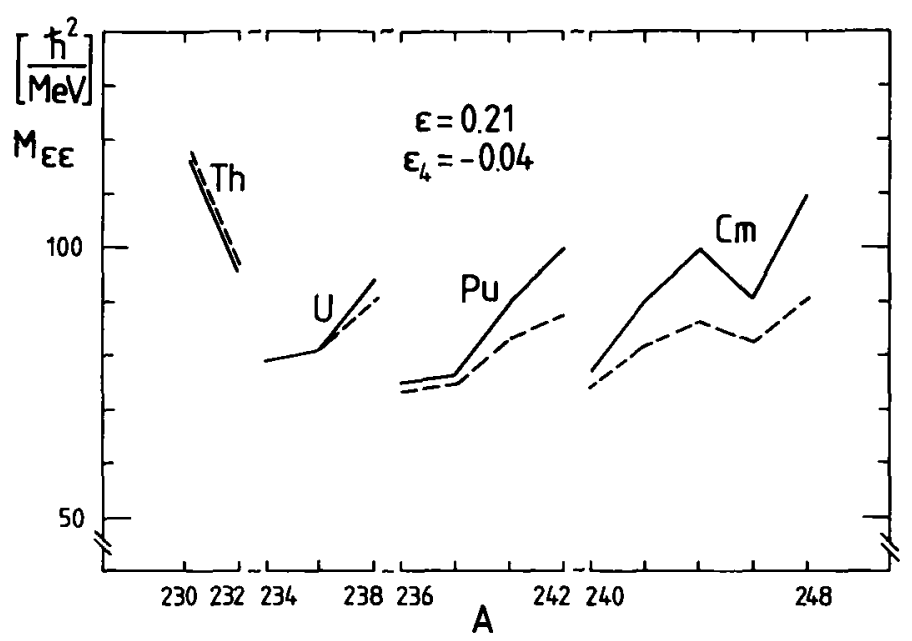

Fig. 7. Quadrupole components of the GCM mass tensor for some actinide nuclei at their average equilibrium deformation $\left(\varepsilon=0.21, \varepsilon_{4}=-0.04\right)$. The solid line joins the points obtained with the constraint of equal deformation of protons and neutrons (eq. (C.4)) and the dashed line corresponds to the isoscalar masses (eq. (C.9)).

both mass tensors are plotted in fig. 6. One can see that the GCM and cranking inertias become closer when $\Omega$ decreases.

The choice of the size $2 \Omega$ of the pairing window is rather crucial in the calculation of the GCM mass parameters. The results are in fact rather sensitive to the choice of $\Omega$. We have assumed throughout the whole paper the same standard pairing window as in ref. ${ }^{18}$ ), although this choice seems to us somewhat arbitrary.

The quadrupole components of the GCM mass tensor for some actinide nuclei are plotted in fig. 7. We have used there two prescriptions for the mass of the composed system, namely eqs. (C.4) and (C.9) corresponding to the "strong coupling" case (same deformation for protons and neutrons) and the isoscalar inertia, respectively. The calculation has been performed for a typical equilibrium deformation $(\varepsilon=0.21$, $\left.\varepsilon_{4}=-0.04\right)$. One can see that both prescriptions give similar values of the collective inertias.

The collective inertias are quantities which can not be directly compared with the experiment. An indirect comparison with experimental data can be only made after extended calculations with the total collective hamiltonian (3.16). But a rough judgement can be obtained by comparing the GCM masses for fissioning nuclei with the phenomenological inertia which reproduces the proper magnitude of fission lifetimes $\left.{ }^{20}\right)$ :

$$
M_{r r}=\mu\left\{1+K \frac{17}{15} \exp \left[\frac{c}{\lambda}\left(\frac{3}{4}-r\right)\right]\right\}
$$




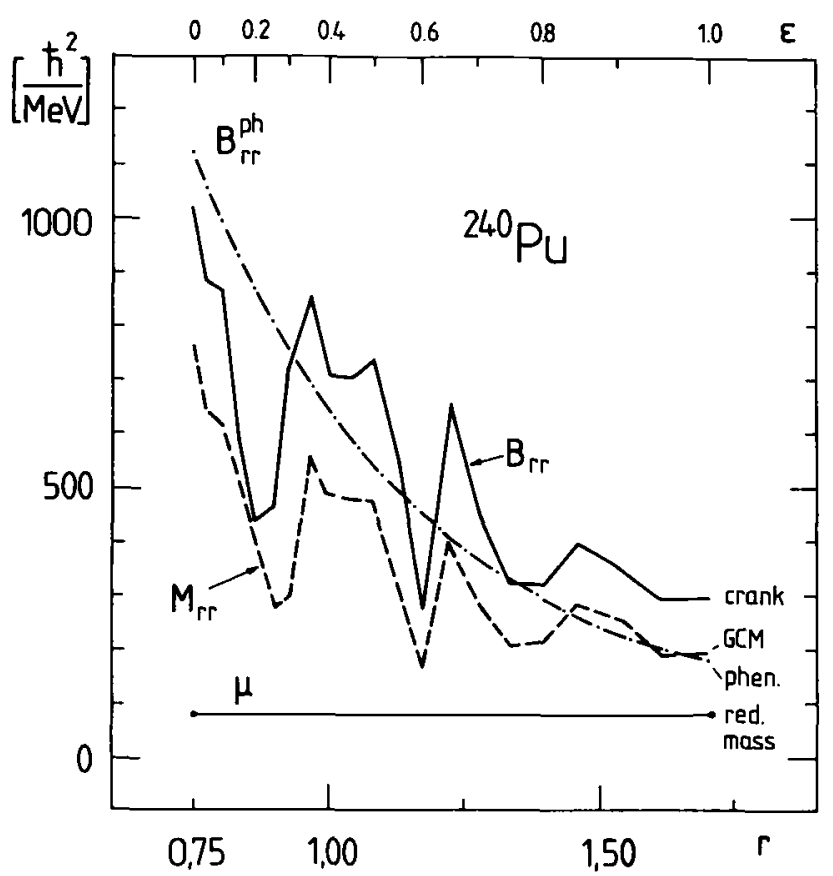

Fig. 8. Cranking (solid line), GCM (dashed line) and phenomenological ${ }^{20}$ ) (dashed-dotted line) masses for ${ }^{240} \mathrm{Pu}$ as functions of the relative distance $(r)$ between the fission fragments.

with $K=11.5$ and $c / \lambda=2.452$. Here $r$ denotes the relative distance between the fission fragments and $\mu$ is the reduced mass.

Three mass parameters: GCM, cranking and $M_{r r}$ eq. (4.2) are plotted in fig. 8 as functions of $r$. The calculation was made along the path $\left(\varepsilon_{24}\right)$ corresponding to the liquid-drop fission valley. We see that for large deformations the GCM mass is closer to the phenomenological inertia than the cranking mass. This suggests that the GCM mass parameter has the proper asymptotic behaviour and is in line with recent results obtained in a two-center shell model. ${ }^{13}$ )

\section{Conclusions}

The following conclusions can be drawn from our investigations.

(i) The zero-point correction energy to the collective potential vanishes as long as we discuss the mean-field plus pairing hamiltonian.

(ii) The mass parameters obtained by the generator coordinate method and gaussian overlap approximation are smaller, on the average by a factor $\frac{2}{3}$, than the cranking model masses.

(iii) GCM and cranking masses show a similar pronounced shell structure in the deformation dependence. 
(iv) The GCM and cranking masses for the elongation mode become close to each other in the diagonal matrix approximation.

(v) The values of the GCM mass parameters depend rather strongly on the choice of the width of the pairing window, even when the average pairing field parameter $(\Delta)$ is constant.

(iv) Both GCM and cranking masses depend strongly on the pairing interaction strength. For large values of the pairing gap parameter $\Delta$ they become close to each other.

(vii) The GCM mass obtained for the compound proton and neutron system with the constraint of equal deformations in the generating proton and neutron functions ("strong coupling case") is very close to the one for the isoscalar (in phase) motion of proton and neutron system.

(viii) The semiempirical mass parameters which reproduce well the spontaneous fission lifetimes ${ }^{20}$ ) for actinides lie between the cranking and GCM inertias when they are drawn as functions of the relative distance between the fission fragments.

We would like to end the paper with some concluding remarks. We want to stress that our investigation should be considered as preliminary. We realize that further improvements have to be added. It seems to us that the most important problems are:

(a) inclusion of the pairing vibration mode $21-23$,

(b) improvement in the treatment of the level-crossing problem in the region of the Fermi surface ${ }^{24}$ ),

(c) use of the generating functions with good particle number instead of ordinary BCS functions,

(d) use of better approximations than the effective mean-field plus pairing hamiltonian.

We believe that these problems ought to be solved in the order given above. They constitute the objects of future investigations.

\section{Appendix A}

We give here the explicit formulae for the second-order expansion coefficient of the reduced energy overlap,

$$
h_{i j}=\sum_{\mu \nu} \frac{\left\langle\nu\left|\partial \hat{H} / \partial a^{i}\right| \mu\right\rangle\left\langle\mu\left|\partial \hat{H} / \partial a^{j}\right| \nu\right\rangle}{E_{\nu}+E_{\mu}}\left(u_{\nu} v_{\mu}+u_{\mu} v_{\nu}\right)^{2}+F_{i j}^{(1)},
$$

the width of the overlap of the generating (BCS) functions,

$$
\gamma_{i j}=\sum_{\mu \nu} \frac{\left\langle\nu\left|\partial \hat{H} / \partial a^{i}\right| \mu\right\rangle\left\langle\mu\left|\partial \hat{H} / \partial a^{j}\right| \nu\right\rangle}{\left(E_{\mu}+E_{\nu}\right)^{2}}\left(u_{\nu} v_{\mu}+u_{\mu} v_{\nu}\right)^{2}+F_{i j}^{(2)},
$$


and the cranking mass tensor,

$$
B_{i j}=2 \sum_{\mu \nu} \frac{\left\langle\nu\left|\partial \hat{H} / \partial a^{i}\right| \mu\right\rangle\left\langle\mu\left|\partial \hat{H} / \partial a^{j}\right| \nu\right\rangle}{\left(E_{\nu}+E_{\mu}\right)^{3}}\left(u_{\nu} v_{\mu}+u_{\mu} v_{\nu}\right)^{2}+F_{i j}^{(3)},
$$

where the tensor $F_{i j}^{(K)}$ contains the terms depending on the derivatives of the gap $(\Delta)$ and the Fermi level $(\lambda)$ on collective coordinates and is equal to

$$
F_{i j}^{(K)}=\sum_{\nu} \frac{\Delta^{2}}{\left(2 E_{\nu}\right)^{K+2}}\left(\beta_{\nu, i} \beta_{\nu, j}-\beta_{\nu, i}\left\langle\nu\left|\frac{\partial \hat{H}}{\partial a^{j}}\right| \nu\right\rangle-\left\langle\nu\left|\frac{\partial \hat{H}}{\partial a^{i}}\right| \nu\right\rangle \beta_{\nu, j}\right)
$$

with

$$
\beta_{\nu, i}=\frac{\partial \lambda}{\partial a^{i}}+\left(e_{\nu}-\lambda\right) \frac{\partial \ln \Delta}{\partial a^{i}} .
$$

The derivatives of $\lambda$ and $\Delta$ can easily be obtained from the BCS gap and particle-number equations:

$$
\begin{aligned}
\frac{\partial \lambda}{\partial a^{i}} & =\frac{\Delta^{2} A_{i} B+C_{i} D}{\Delta^{2} B^{2}+D^{2}}, \\
\frac{\partial \ln \Delta}{\partial a^{i}} & =\frac{A_{i} D-B C_{i}}{\Delta^{2} B^{2}+D^{2}},
\end{aligned}
$$

where

$$
\begin{array}{ll}
A_{i}=\sum_{\nu} \frac{\left\langle\nu\left|\partial \hat{H} / \partial a^{i}\right| \nu\right\rangle}{E_{\nu}^{3}}, & B=\sum_{\nu} \frac{1}{E_{\nu}^{3}}, \\
C_{i}=\sum_{\nu} \frac{\left\langle\nu\left|\partial \hat{H} / \partial a^{i}\right| \nu\right\rangle\left(e_{\nu}-\lambda\right)}{E_{\nu}^{3}}, & D=\sum_{\nu} \frac{e_{\nu}-\lambda}{E_{\nu}^{3}} .
\end{array}
$$

\section{Appendix B}

We shall discuss here in some more detail the approximation made in this paper by using a phenomenological, deformation-dependent shell-model hamiltonian. Ideally, one wants to use a many-body hamiltonian of the form

$$
\hat{H}=\sum_{i} \hat{t}_{i}+\frac{1}{2} \sum_{i j} V(i, j),
$$

where $V(i, j)$ is some effective nucleon-nucleon interaction (e.g. a $G$-matrix or a phenomenological Skyrme-type interaction). The optimal single-particle basis for construction of the generator coordinate wave functions is the Hartree-Fock (HF) basis derived from $\hat{H}$, eq. (B.1). However, in order to obtain these single-particle states as functions of some deformation parameters (i.e. the generator coordinates) $q_{\mu}$, one is forced to perform the HF variational procedure including external 
constraints, viz.

$$
\delta\left\langle\hat{H}-\sum_{\mu} \lambda_{\mu} \hat{q}_{\mu}\right\rangle=0
$$

(adding the usual Lagrange multipliers for the normalisation of the s.p. states). In eq. (B.2), $\lambda_{\mu}$ are Lagrange multipliers and $\hat{q}_{\mu}$ are some suitable (one-body) operators (e.g., but not necessarily, multipole operators) chosen such that their expectation values give the desired deformation parameters:

$$
\left\langle\hat{q}_{\mu}\right\rangle=q_{\mu} \text {. }
$$

The variation in eq. (B.2) leads to the HF equations

$$
\left[\hat{T}+\mathscr{U}_{\mathrm{HF}}-\sum_{\mu} \lambda_{\mu} \hat{q}_{\mu}\right] \phi_{i}=\varepsilon_{i} \phi_{i},
$$

where the (generally nonlocal) mean field $\mathscr{U}_{\mathrm{HF}}$ as well as the $\varepsilon_{i}$ and $\phi_{i}$ depend on the $q_{\mu}$. The s.p. wave functions are then used to build the generator coordinate wave functions in the form of Slater determinants:

$$
|q\rangle=\frac{1}{\sqrt{A !}} \operatorname{det}\left|\phi_{i}\left(r_{j} ; q\right)\right| .
$$

In the often-used phenomenological approach one replaces the mean HF field, including the constraints, by a deformation-dependent shell-model potential:

$$
\hat{\mathscr{U}}_{\mathrm{HF}}(q)-\sum_{\mu} \lambda_{\mu} \hat{q}_{\mu} \rightarrow \mathscr{U}_{\mathrm{SM}}(\varepsilon),
$$

where $\varepsilon$ stands for a set $\left\{\varepsilon_{\mu}\right\}$ of deformation parameters. It may now be argued that this de facto inclusion of the external constraints gives some spurious contributions to the collective potential energy and inertial parameters, since these must be calculated from the unconstrained hamiltonian $\hat{H}$, eq. (B1), and that one therefore should rather use

$$
\tilde{\mathscr{U}}=\mathscr{U}_{\mathrm{SM}}(\varepsilon)+\sum_{\mu} \lambda_{\mu} \hat{q}_{\mu}
$$

However, we shall presently show that the replacement of $\mathscr{U}_{\text {SM }}(\varepsilon)$ by $\overline{\mathscr{U}}$ does not affect the physically observable quantities. For that, we expand the deformationdependent part of $\mathscr{U}_{\mathrm{SM}}(\varepsilon)$ in terms of the (multipole) operators $\hat{q}_{\mu}$ :

$$
\mathscr{U}_{\mathrm{SM}}(\varepsilon)=\mathscr{U}_{\mathrm{SM}}(0)+\sum_{\mu} \alpha_{\mu}(\varepsilon) \hat{q}_{\mu}
$$

where $\alpha_{\mu}(\varepsilon)$ will be unique functions of the deformation parameters $\varepsilon_{p}$. On the other hand, the Lagrange multipliers $\lambda_{\mu}$ in eqs. (B.2) and (B.4) can also be expressed in terms of the $\varepsilon_{\nu}$. They are given from the HF deformation energy by

$$
\lambda_{\mu}(q)=\frac{\mathrm{d}}{\mathrm{d} q^{\mu}}\langle q|\hat{H}| q\rangle
$$


The $q_{\mu}$ in turn can be related to the $\varepsilon_{\mu}$ through the expectation values of $\hat{q}_{\mu}$ in the shell-model states:

$$
q_{\mu}(\varepsilon)=\left\langle\varepsilon\left|\hat{q}_{\mu}\right| \varepsilon\right\rangle .
$$

Combining eqs. (B.7) and (B.8), we can thus write

$$
\tilde{\mathscr{U}}=\mathscr{U}_{\mathrm{SM}}(0)+\sum_{\mu}\left[\alpha_{\mu}(\varepsilon)+\lambda_{\mu}(\varepsilon)\right] \hat{q}_{\mu} .
$$

We now claim that in practical cases it should always be possible to define a new set of deformation parameters $\left\{\tilde{\varepsilon}_{\nu}\right\}$ so that

$$
\alpha_{\mu}(\varepsilon)+\lambda_{\mu}(\varepsilon)=\alpha_{\mu}(\tilde{\varepsilon}) .
$$

Note that the $\tilde{\varepsilon}_{v}$ will not differ much from the $\varepsilon_{v}$; they are, in fact, identical in all stationary points of the deformation energy surface since the $\lambda_{\mu}$ eq. (B.9) vanish there. Thus, inclusion of the external constraints in $\tilde{\mathscr{U}}$ will amount to a coordinate transformation

$$
\varepsilon_{\nu} \rightarrow \tilde{\varepsilon}_{\nu}
$$

so that

$$
\tilde{\mathscr{U}}=\mathscr{U}_{\mathrm{SM}}(\tilde{\varepsilon}) .
$$

Since the transformation eq. (B.13) has a series of fix-points (the zero of $\lambda_{\mu}$ corresponding to minima and saddle points in the deformation energy surface), it can be expected to be well-behaved. Now, all physical observables obtained from a dynamical calculation - such as e.g. vibrational frequencies or fission lifetimes depending on action integrals - are invariant under coordinate transformations in collective space, such as eq. (B.13), as becomes evident from the covariant formalism used in sect. 3. Therefore they will not be affected by the inclusion of the external constraints, if the $\tilde{\varepsilon}_{v}$ are consistently used as generator coordinates in the GCM calculations along with $\tilde{\mathscr{U}}(\tilde{\varepsilon})$. (Different intermediate results for mass parameters and collective potential energies are, of course, obtained if the original coordinates $\varepsilon_{\nu}$ are kept and the external constraints included in $\tilde{\mathscr{U}}$. The final results of the collective dynamics, however, have to be the same.)

\section{Appendix C}

THE TOTAL MASS FOR A COMPOSITE NEUTRON AND PROTON SYSTEM

For nuclear collective motion we have to consider a system of protons and neutrons. We assume that the total hamiltonian is a sum of those for protons and neutrons separately:

$$
\hat{H}=\hat{H}_{\mathrm{p}}+\hat{H}_{\mathrm{n}} .
$$

The only coupling between the two kinds of nucleons is due to the common 
deformation parameters $q$ :

$$
|\psi\rangle=\int \mathrm{d} q f(q)|q\rangle_{\mathrm{n}}|q\rangle_{\mathrm{p}}
$$

In analogy to the cranking mass we define the GCM mass tensor as the inverse matrix of the contravariant tensor $\mathscr{M}^{-1}=\left\{\left(\mathscr{M}^{-1}\right)^{i j}\right\}$ contained in the collective hamiltonian (3.16). From eq. (3.19) one can immediately see that with the wave function (C.2) the total covariant inverse mass tensor is a sum of those for neutrons and protons separately:

$$
\left(\mathscr{M}^{-1}\right)_{\mathrm{cov}}=\left(\mathscr{M}_{\mathrm{n}}^{-1}\right)_{\mathrm{cov}}+\left(\mathscr{M}_{\mathrm{p}}^{-1}\right)_{\mathrm{cov}} .
$$

The total metric tensor $\gamma$ is also a sum of the metric tensors for the neutron $\stackrel{n}{\gamma}$ and proton $\stackrel{\gamma}{\gamma}$ subsystems treated independently. For only one collective variable, eq. (C.3) can be rewritten in the simple form

$$
M=\gamma^{2} \frac{\mathscr{M}_{\mathrm{n}} \mathscr{M}_{\mathrm{p}}}{\gamma^{2} \mathscr{M}_{\mathrm{p}}+\gamma^{2} \mathscr{M}_{\mathrm{n}}},
$$

where

$$
\stackrel{k}{\gamma}={ }_{k}\left\langle q\left|\frac{\bar{\partial}}{\partial q} \frac{\vec{\partial}}{\partial q}\right| q\right\rangle_{k}
$$

with $k=\mathrm{n}$ or $\mathrm{p}$ and the total metric tensor is $\gamma=\stackrel{\mathrm{n}}{\gamma}+\stackrel{\mathrm{p}}{\gamma}$.

Another possibility to obtain the total mass is to start from the "two-dimensional

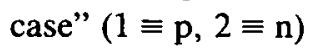

$$
|\psi\rangle=\int \mathrm{d} q^{1} \mathrm{~d} q^{2} f\left(q^{1}, q^{2}\right)\left|q^{1}\right\rangle\left|q^{2}\right\rangle
$$

For simplicity, we use in the following only one collective variable for the proton and one for the neutron subsystem. In the next step we introduce new variables

$$
x^{1}=\frac{1}{2}\left(q^{1}+q^{2}\right), \quad x^{2}=\frac{1}{2}\left(q^{1}-q^{2}\right) .
$$

In these variables the metric tensor is no longer diagonal and can be expressed as

$$
\gamma_{11}=\gamma_{22}=\stackrel{n}{\gamma}+\stackrel{p}{\gamma}, \quad \gamma_{12}=\gamma_{21}=\stackrel{n}{\gamma}-\stackrel{p}{\gamma}
$$

The contravariant inverse mass tensor can be directly obtained from eq. (3.17):

$$
\begin{aligned}
& \left(\mathscr{M}^{-1}\right)^{x x}=\frac{1}{4}\left[\left(\mathscr{M}_{\mathrm{n}}^{-1}\right)+\left(\mathscr{M}_{\mathrm{p}}^{-1}\right)\right]=\left(\mathscr{M}^{-1}\right)^{y y}, \\
& \left(\mathscr{M}^{-1}\right)^{x y}=\frac{1}{4}\left[\left(\mathscr{M}_{\mathrm{n}}^{-1}\right)-\left(\mathscr{M}_{\mathrm{p}}^{-1}\right)\right] .
\end{aligned}
$$

The $x x$ component corresponds to the isoscalar mass (protons and neutrons move in phase) and the $y y$ component relates to the isovector mode. Because in the 
hamiltonian (C.1) no interaction between protons and neutrons is assumed, both masses are equal. It is expected that for low excitation energy only a collective motion in $x$-direction is realized. Then the total mass of the motion can be calculated from the $x x$ component of $\mathscr{H}^{-1}$ :

$$
M=\left[\left(\mathscr{M}^{-1}\right)^{x x}\right]=\frac{4 \mathscr{M}_{\mathrm{n}} \mathscr{M}_{\mathrm{p}}}{\mathscr{M}_{\mathrm{n}}+\mathscr{M}_{\mathrm{p}}} .
$$

In practice, expressions (C.4) and (C.9) often give similar values of the total mass. Throughout this paper we use expression (C.4) for the masses of the total nucleus.

\section{References}

1) D.R. Inglis, Phys. Rev. 96 (1954) 1059; 103 (1956) 1786; S.T. Belyeav, Mat. Fys. Medd. Dan. Vid. Selsk. 31, No. 11 (1959); Nucl. Phys. 24 (1961) 222

2) D.R. Bès, Mat. Fys. Medd. Dan. Vid. Selsk. 33, No. 2 (1961)

3) A. Sobiczewski, Z. Szymański. S. Wycech, S.G. Nilsson, J.R. Nix, X.F. Tsang, C. Gustafson, P. Möller and B. Nilsson, Nucl. Phys. A131 (1969) 67

4) M. Brack, J. Damgaard, A.S. Jensen, H.C. Pauli, V.M. Strutinsky, and C.Y. Wong. Rev. Mod. Phys. 44 (1972) 320

5) S.G. Rohozinski, J. Dobaczewski, B. Nerlo-Pomorska. K. Pomorski and J. Srebrny, Nucl. Phys. A292 (1977) 66

6) H. Hofmann, Z. Phys. 250 (1977) 14

7) D.M. Brink and A. Weiguny, Nucl. Phys. A120 (1968) 59

8) J.J. Griffin and J.A. Wheeler, Phys. Rev. 108 (1957) 311;

D.L. Hill and J.A. Wheeler, Phys. Rev. 89 (1953) 1102

9) B. Giraud and B. Grammaticos, Nucl. Phys. A233 (1974) 373: A255 (1975) 141: A330 (1979) 40

10) N. Onishi and T. Une, Prog. Theor. Phys. 53 (1975) 504

11) P.G. Reinhard, Nucl. Phys. A252 (1975) 120, A261 (1976) 291; A306 (1978) 19;

P.G. Reinhard, F. Grümmer and K. Goeke, Z. Phys. A317 (1984) 339

12) C.W. Wong, Nucl. Phys. Al47 (1970) 545;

L. Lathouwers, Ann. of Phys. 102 (1976) 347;

A.F.R. de Toledo Piza et al., Phys. Rev. C15 (1977) 1477

13) C. Fiolhais and R.M. Dreizler, Nucl. Phys. A393 (1983) 205

14) S.J. Wang, W. Cassing and W. Nörenberg, Phys. Lett. 131B (1983) 265

15) P. Ring and P. Schuck. The nuclear many-body problem (Springer, New York, 1980)

16) A. Góżdż, Phys. Lett. 152B (1985) 281

17) K. Pomorski, Acta Phys. Pol. B7 (1976) 595

18) S.G. Nilsson, C.F. Tsang, A. Sobiczewski, Z. Szymanski, S. Wycech, C. Gustafson, I.L. Lamm, P. Möller and B. Nilsson, Nucl. Phys. A131 (1969) 1

19) K. Böning, A. Sobiczewski and K. Pomorski, Acta. Phys. Pol. B, in print

20) J. Randrup, S.E. Larsson, P. Möller, S.G. Nilsson, K. Pomorski and A. Sobiczewski, Phys. Rev. C13 (1976) 394

21) D.R. Bès, A. Broglia, R.P.J. Perazzo and K. Kumar, Nucl. Phys. A143 (1970) 1

22) K. Pomorski, K. Zajac, B. Nerlo-Pomorska and S.G. Rohoziński, Proc. XIX Winter School on physics, Zakopane, Poland, 3-15 April 1984

23) A. Góżdż et al., Nucl. Phys. A442 (1985) 50

24) A. Góźdż, W. Werner and M. Brack, Z. Phys. A319 (1984) 159 